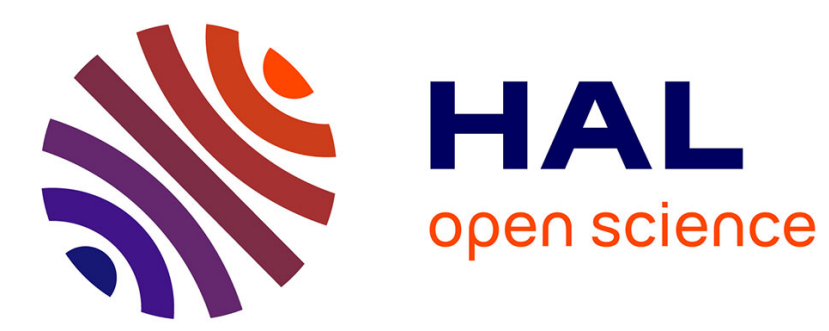

\title{
SUBSPACE SIGNAL-TO-NOISE RATIO MAXIMIZATION: THE CONSTRAINED STOCHASTIC MATCHED FILTER
}

Bruno Borloz, Bernard Xerri

\section{- To cite this version:}

Bruno Borloz, Bernard Xerri. SUBSPACE SIGNAL-TO-NOISE RATIO MAXIMIZATION: THE CONSTRAINED STOCHASTIC MATCHED FILTER. Eighth International Symposium on Signal Processing and Its Applications, 2005., 2005, Sydney, France. 10.1109/ISSPA.2005.1581043 . hal01823750

\section{HAL Id: hal-01823750 \\ https://hal-amu.archives-ouvertes.fr/hal-01823750}

Submitted on 29 Jun 2018

HAL is a multi-disciplinary open access archive for the deposit and dissemination of scientific research documents, whether they are published or not. The documents may come from teaching and research institutions in France or abroad, or from public or private research centers.
L'archive ouverte pluridisciplinaire HAL, est destinée au dépôt et à la diffusion de documents scientifiques de niveau recherche, publiés ou non, émanant des établissements d'enseignement et de recherche français ou étrangers, des laboratoires publics ou privés. 


\title{
SUBSPACE SIGNAL-TO-NOISE RATIO MAXIMIZATION: THE CONSTRAINED STOCHASTIC MATCHED FILTER
}

\author{
Bruno Borloz - Bernard Xerri \\ Université du Sud Toulon-Var \\ Laboratoire STIC/GESSY BP 56, 83162 La Valette du Var Cedex (FRANCE) \\ borloz, xerrieuniv-tln.fr
}

\begin{abstract}
This paper deals with signal-to-noise ratio (SNR) maximization in a subspace. This criterion can be applied to estimation or detection of a stochastic signals embedded in additive noise. Both signal and noise are considered to be realizations of random functions whose statistics are assumed known up to second-order. The proposed method leads to a filter we call "constrained stochastic matched filter" (CSMF), optimal in the sense that it maximizes the SNR in a subspace whose dimension is chosen a priori. It is an extension of the stochastic matched filter (SMF), itself an extension of the matched filter.
\end{abstract}

\section{INTRODUCTION}

The study presented in this paper is concerned with detection or estimation of stochastic signals embedded in noise. The available information is the covariance matrices of signal and noise.

When key quantities like the probability density function of the signals studied are not available, it is legitimate to try to use methods based on energetic criterion. Hence the output SNR maximization, i.e. after a filtering operation, seems a natural way to attack problems of detection or estimation. It requires the knowledge of second-order statistics of both signal and noise.

In this scope, several approaches can be considered, two main of which are the matched filter [1][2][3] and derived approaches [4][5][6][7][8] and classification techniques as, for example, the Fisher linear discriminant analysis applied to the particular case of a two-classes problem [9][10]. The method proposed is derived from the first approach in the sense that it shares the same philosophical framework: a SNR maximization.

For example, it is well known that the linear filter that has maximum output SNR at some instant of time when input is a deterministic signal embedded in an additive corrupting random noise is the matched filter [3]. When noise is Gaussian, the output of this filter provides a sufficient statistic for any Likelihood Ratio Test for detection of presence of the input signal. A complete specification of signal and second-order statistics of noise is necessary to derive the corresponding matched filter.

Derived methods have been proposed to face this problem when the signal to detect is no longer perfectly known, due to channel nonlinearities, timing jitter, nonstationarities, modeling uncertainties, etc. For example, the minimax robust matched-filter [4][5] optimizes the worst-case performance over an uncertainty class. The problem of detecting a transmitted signal when one of $M$ known signals is transmitted (e.g. in digital communications [6][7]) leads, when the additive noise is white and gaussian, to an optimal detector consisting in a bank of matched filters followed by a detector which chooses as the detected signal the one of which the output is maximal.

The stochastic matched filter (SMF) was introduced to take into consideration the problem of detecting a stochastic signal whose second-order statistics are known [8]. It extends the matched filter approach because it gives an optimal filter in the sense that it makes maximum the output SNR expressed in the form of Rayleigh's quotient. This problem is a generalized eigenvalues one with suitable matrices, say the covariance matrices of signal and noise. Filtering consists of a projection onto an optimal subspace with direction of the eigenvector associated to the largest eigenvalue, which indeed can be interpreted as the output SNR.

A natural extension is to take into account more eigenvectors deduced from this method: this could be called Extended SMF (ESMF). Actually, it has been shown [8] that other eigenvectors (associated to the largest eigenvalues) can statistically contribute to improve detection.

The method proposed in this paper is a natural extension of the SMF method, naturally inferred from the remark above concerning a projection onto a subspace of dimension two or higher; it aim is to make maximum the output SNR in an aptly chosen subspace. This is why we call this optimal filter 'Constrained Stochastic Matched Filter' (CSMF). It takes place in the class of the subspace projection methods whose interest is increasing [11][12][13]. 


\section{NOTATIONS AND CLASSICAL METHODS}

Throughout this paper each signal is discrete and then represented by a vector $\mathbf{s}$ of $E_{N}$. Moreover, we will suppose that $\mathbf{s}$ is zero mean.

\subsection{Notations and basic developments}

A $N$-dimensional random vector $\mathbf{s}$ can be expressed as follows:

$$
\mathbf{s}=\sum_{i=1}^{N} \alpha_{i} \mathbf{v}_{i}=\mathbf{V} \alpha
$$

where the $\alpha_{i}$ are random variables, the $\left\{\mathbf{v}_{i}\right\}$ linearly independent unit vectors, $\mathbf{V}=\left[\mathbf{v}_{1} \ldots \mathbf{v}_{N}\right]$ and $\alpha=\left[\alpha_{1} \ldots \alpha_{N}\right]^{\top}$. As we are interested in calculating the power of signals in a $p$-dimension subspace $E_{p}$, quantity that must not depend on the basis used to described this subspace, we can without loss of generality assume that the $\left\{\mathbf{v}_{i}\right\}$ are orthonormal.

Covariance matrix $\mathbf{A}=\mathrm{E}\left(\mathbf{s s}^{\top}\right)$ and the power $P_{s}$, after a projection onto the subspace spanned by the vectors $\mathbf{V}_{p}=$ $\left[\mathbf{v}_{1}, \mathbf{v}_{2}, \ldots \mathbf{v}_{p}\right]$ are related by:

$$
P_{s}=\operatorname{tr}\left(\mathbf{V}_{p}^{\top} \mathbf{A} \mathbf{V}_{p}\right)=\sum_{i=1}^{p} \mathbf{v}_{i}^{\top} \mathbf{A} \mathbf{v}_{i} .
$$

Similar calculations can be performed for the noise vector $\mathbf{n}$ with covariance matrix $\mathbf{B}$.

\subsection{The matched filter}

If $\mathbf{s}$ is a known deterministic signal, the objective is to find a linear filter (a vector) $\mathbf{h}$ so as to maximize the output SNR defined by:

$$
\frac{\left(\mathbf{s}^{\top} \mathbf{h}\right)^{2}}{\mathbf{h}^{\top} \mathbf{B h}}=\frac{\mathbf{h}^{\top} \mathbf{s} \mathbf{S}^{\top} \mathbf{h}}{\mathbf{h}^{\top} \mathbf{B h}} .
$$

It is well established that the optimal filter is a correlation filter called the "matched filter", $\mathbf{h}=\mathbf{B}^{-1} \mathbf{s}$ and that the output SNR is $\mathbf{S}^{\top} \mathbf{B}^{-1} \mathbf{S}$.

\subsection{The stochastic matched filter}

This approach was developed [8] to take into consideration the case where $\mathbf{s}$ is a stochastic signal uncorrelated with noise in lieu of the deterministic one. The output SNR can be written like a Rayleigh quotient:

$$
\frac{\mathbf{h}^{\top} \boldsymbol{\Gamma}_{s} \mathbf{h}}{\mathbf{h}^{\top} \mathbf{B h}}
$$

where $\mathbf{h}$ is the filter, i.e. a $N$-dimensional vector. This is a stationary-value problem which can be resolved by solving the so-called generalized eigenvalue problem:

$$
\boldsymbol{\Gamma}_{s} \mathbf{h}=\lambda \mathbf{B h} .
$$

The maximal SNR $\rho_{\max }$ is obtained for the filter $\mathbf{h}=\mathbf{u}_{1}$, say the eigenvector associated with the largest eigenvalue $\lambda_{1}$ of $\mathbf{B}^{-1} \mathbf{A}$; then $\rho_{\max }=\lambda_{1}$ : this filtering comes down to projecting the signal onto a one-dimensional subspace, which was the initial objective. Calculations lead to the eigenvectors of $\mathbf{B}^{-1} \mathbf{A}$, one of them being optimal. Using all the eigenvectors of $\mathbf{B}^{-1} \mathbf{A}$, we perform a simultaneous diagonalization of $\mathbf{A}$ and $\mathbf{B}$.

Made from intuitive arguments, experiments show that the use of more than one eigenvector could improve the detection of the signal of interest. But even if this basis maximizes the SNR in a one-dimensional subspace, there is no reason that it would do it also in a $p$-dimensional subspace. This is why the approach of the constrained stochastic matched filter has been developed.

\section{THE CONSTRAINED STOCHASTIC MATCHED FILTER}

There is no reason why the eigenvectors of $\mathbf{B}^{-1} \mathbf{A}$ should span a subspace of dimension $p>1$ where the SNR is maximum. We propose here to define and find such a subspace whose dimension is set a priori.

\subsection{SNR in a $p$-dimensional subspace}

From (1) it comes that the output SNR after projection onto $E_{p}$ spanned by $p$ orthonormal vectors $\left\{\mathbf{v}_{i}\right\}$ is:

$$
\rho=\frac{\operatorname{tr}\left(\mathbf{V}_{p}^{\top} \mathbf{A} \mathbf{V}_{p}\right)}{\operatorname{tr}\left(\mathbf{V}_{p}^{\top} \mathbf{B} \mathbf{V}_{p}\right)}=\frac{\sum_{i=1}^{p} \mathbf{v}_{i}^{\top} \mathbf{A} \mathbf{v}_{i}}{\sum_{i=1}^{p} \mathbf{v}_{i}^{\top} \mathbf{B} \mathbf{v}_{i}}
$$

Our objective is to maximize this ratio. That means, $p$ beeing chosen a priori, find the $p$-dimensional subspace $E_{p}$ in which this value is maximum: this optimal subspace will be noted $E_{p}^{*}$.

Without loss of generality, we can state that $\operatorname{tr} \mathbf{A}=\operatorname{tr} \mathbf{B}=1$. That means that the initial SNR $\frac{\operatorname{tr} \mathbf{A}}{t r \mathbf{B}}$ is 1 and then $\rho$ appears to be a gain on the SNR.

\subsection{Properties of the optimal subspace $E_{p}^{*}$}

$p$ is given and the unknowns of the problem are the SNR $\rho$ defined by (2) and the $\mathbf{v}_{i}$ 's which must be calculated to maximize $\rho$. The constraints can be expressed by $\mathbf{v}_{i}^{\top} \mathbf{v}_{j}=$ $\delta_{i j}$. This optimization problem with constraints is usually solved with a Lagrange multipliers method. Hence let's define the function:

$$
L\left(\mathbf{v}_{i}, \omega_{i j}\right)=\rho+\sum_{i=1}^{p} \sum_{j=1}^{p} \omega_{i j}\left(\mathbf{v}_{i}^{\top} \mathbf{v}_{j}-\delta_{i j}\right)
$$


or

$$
L\left(\mathbf{V}_{p}, \boldsymbol{\Omega}\right)=\frac{\operatorname{tr}\left(\mathbf{V}_{p}^{\top} \mathbf{A} \mathbf{V}_{p}\right)}{\operatorname{tr}\left(\mathbf{V}_{p}^{\top} \mathbf{B} \mathbf{V}_{p}\right)}+\operatorname{tr}\left(\boldsymbol{\Omega}\left(\mathbf{V}_{p}^{\top} \mathbf{V}_{p}-\mathbf{I}_{p}\right)\right),
$$

where $\boldsymbol{\Omega} \equiv\left[\omega_{i j}\right]$ is a $p \times p$ symmetric matrix. This value is maximum when $\frac{\partial L}{\partial \mathbf{v}_{p}}=0$. Calculation leads to:

$$
(\mathbf{A}-\rho \mathbf{B}) \mathbf{V}_{p}=\mathbf{V}_{p} \boldsymbol{\Omega}_{0}
$$

where $\boldsymbol{\Omega}_{0}$ is a $p \times p$ real symmetric matrix. There exist a $p \times p$ real orthogonal matrix $\Phi$ and a $p \times p$ real diagonal matrix such as $\Delta$ such that $\boldsymbol{\Omega}_{0}=\boldsymbol{\Phi} \Delta \boldsymbol{\Phi}^{\top}$. Then equation (3) becomes:

$$
(\mathbf{A}-\rho \mathbf{B}) \mathbf{V}_{p} \boldsymbol{\Phi}=\mathbf{V}_{p} \boldsymbol{\Phi} \Delta .
$$

As $\boldsymbol{\Phi}$ is invertible, $\mathbf{V}_{p}$ and $\mathbf{V}_{p} \boldsymbol{\Phi}$ span the same subspace. Then $E_{p}^{*}$ is spanned by $p$ vectors $\mathbf{v}_{i}$ among the $N$ satisfying the eigenvalue equation (4).

$(\mathbf{A}-\rho \mathbf{B})$ is a symmetric matrix depending on $\rho$, diagonalizable, of unitary eigenvectors matrix $\mathbf{T}=\left[\mathbf{t}_{1} \ldots \mathbf{t}_{N}\right]$ :

$$
(\mathbf{A}-\rho \mathbf{B}) \mathbf{t}_{i}=\mu_{i} \mathbf{t}_{i} \quad \forall i=1, \ldots N .
$$

As $\rho$ is unknown, this is not a common eigenvalue problem. Let's choose a subset $I$ of $\{1, \ldots N\}$ satisfying $\operatorname{card}(I)=p$; then

$$
\frac{\sum_{i \in I} \mathbf{t}_{i}^{\top} \mathbf{A} \mathbf{t}_{i}}{\sum_{i \in I} \mathbf{t}_{i}^{\top} \mathbf{B} \mathbf{t}_{i}}-\rho=\frac{\sum_{i \in I} \mu_{i}}{\sum_{i \in I} \mathbf{t}_{i}^{\top} \mathbf{B} \mathbf{t}_{i}} .
$$

In the subspace $E_{p}^{*}$ the left expression is null. Hence the $p$ optimal vectors $\left\{\mathbf{t}_{i}\right\}_{\{i \in I\}}$ which span $E_{p}^{*}$ are those for which:

$$
\sum_{i \in I} \mu_{i}=0
$$

The $\mathbf{t}_{i}, \mu_{i}$ and $\rho$ are unknown. In fact, the solution is directly calculable only for the particular cases $p=1$ and $p=N-1$. An algorithm to reach the solution is proposed in section 3.3.

\section{Remarks}

1. If $p=1$, the eigenvalue $\mu_{1}$ to take into account is null. Hence, $(\mathbf{A}-\rho \mathbf{B}) \mathbf{t}_{1}=0$ i.e. $\quad \mathbf{A t}_{1}=\rho \mathbf{B} \mathbf{t}_{1} . \quad \rho$ is the largest eigenvalue of $\mathbf{B}^{-1} \mathbf{A}$ and $\mathbf{t}_{1}$ its associated eigenvector. Naturally we find in this case the SMF.

2. From simple examples, it is possible to infer two significant conclusions. First, the optimal subspace $E_{p}^{*}$ is not necessarily spanned by the eigenvectors of $\mathbf{B}^{-1} \mathbf{A}$, and even though this is the case, the eigenvectors are not necessarily associated with the largest eigenvalues. Secondly, it is no use thinking of a recursive formulation on $p$ to find $E_{p}^{*}$.

\section{Relations on the eigenvectors and eigenvalues}

A study of the behavior of the $\mu_{i}$ w.r.t. $\rho$ shows that $\mu_{i}(0)=$ $\mu_{i}^{A}>0$ (eigenvalue of $\mathbf{A}$ ) and for $\rho \rightarrow+\infty \mu_{i}(\rho) \rightarrow$ $-\rho \mu_{i}^{B} \rightarrow-\infty$. In fact,

$$
\forall i, \quad \frac{\partial \mu_{i}}{\partial \rho}=-\mathbf{t}_{i}^{\top} \mathbf{B} \mathbf{t}_{i}<0
$$

the curves $\mu_{i}(\rho)$ are decreasing with a linear slope at infinity.

\subsection{Algorithm to find $E_{p}^{*}$}

\subsubsection{Presentation}

Let's take an initial value of $\rho$ (a reasonable value is the largest eigenvalue of $\mathbf{B}^{-1} \mathbf{A}$ ) denoted $\rho_{0}$; we obtain the matrix $\mathbf{A}-\rho_{0} \mathbf{B}$. One can calculate the $N$ eigenvectors of it, $\mathbf{t}_{i}\left(\rho_{0}\right)$, and choose among them the $p$ ones for which

$$
\rho_{1}=\frac{\sum_{i \in I_{0}} \mathbf{t}_{i}^{\top}\left(\rho_{0}\right) \mathbf{A} \mathbf{t}_{i}\left(\rho_{0}\right)}{\sum_{i \in I_{0}} \mathbf{t}_{i}^{\top}\left(\rho_{0}\right) \mathbf{B} \mathbf{t}_{i}\left(\rho_{0}\right)}
$$

is maximum. These $p$ vectors span a subspace $E_{p}^{(0)}$.

At last, one can calculate $\mathbf{A}-\rho_{1} \mathbf{B}, I_{1}$ and the new subspace $E_{p}^{(1)}$ and iterate the process until $\Delta \rho_{n}=\rho_{n+1}-\rho_{n}<\varepsilon$. It remains to prove that this algorithm converges to the good solution $\rho_{\max }$.

\subsubsection{Study of convergence}

Noting $\mu_{i, n}=\mu_{i}\left(\rho_{n}\right), \mathbf{t}_{i, n}=\mathbf{t}_{i}\left(\rho_{n}\right)$ and for the optimal values $\mu_{i}^{*}=\mu_{i}\left(\rho_{\max }\right)$, let's demonstrate that the algorithm proposed converges when starting near from the solution. We know that for $\rho_{\max }$, there exists a subset $I^{*} \subset\{1, \ldots N\}$ such that $\operatorname{card}(I)=p$ and $\sum_{i \in I^{*}} \mu_{i}^{*}=0$.

At step $n$, from (5) and (7),(6), the variation of $\rho$ is:

$$
\Delta \rho_{n} \triangleq \rho_{n+1}-\rho_{n}=\frac{\sum_{i \in I_{n}} \mu_{i, n}}{\sum_{i \in I_{n}} \mathbf{t}_{i, n}^{\top} \mathbf{B} \mathbf{t}_{i, n}}=\frac{\sum_{i \in I_{n}} \mu_{i, n}}{-\sum_{i \in I_{n}} \frac{\partial \mu_{i, n}}{\partial \rho}} .
$$

Note that if $\sum_{i \in I_{n}} \mu_{i, n}=\sum_{i \in I} \mu_{i}^{*}=0$, then $\Delta \rho_{n}=0 . \rho_{\max }$ is an attractive fixed-point of the algorithm.

If we note $f(\rho)=\sum_{i \in I} \mu_{i}$, this equation can be written

$$
\Delta \rho_{n}=\rho_{n+1}-\rho_{n}=-\frac{f\left(\rho_{n}\right)}{f^{\prime}\left(\rho_{n}\right)} .
$$

This algorithm is nothing else than the Newton-Raphson method applied to $f$ twice differentiable, which owns a zero $\left(\rho_{\max }\right)$ with $f\left(\rho_{\max }\right) \neq 0$. It is known that such an algorithm converges with a quadratic speed. 


\subsection{3. simulation}

We present here a simulation. Each realization is made up of twenty one samples. We build ROC curves, for the two methods, with 410 realizations. Signal and noise are zero mean. Covariance matrices are estimated with 100 realizations. The CSMF is as good as ESMF or better.

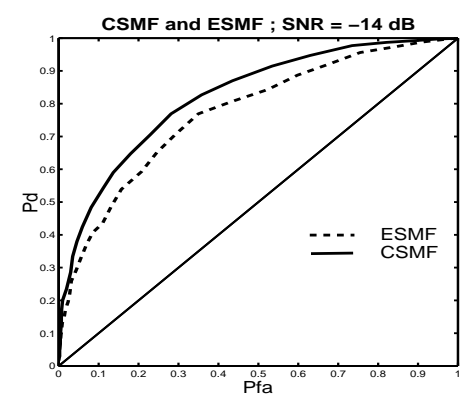

Figure 1: COR curves for CSMF and ESMF : SNR -14 dB

\section{CONCLUSION}

The method proposed in this paper takes place in the set of methods of decomposition of signals on adapted basis and of subspace-based methods.

When trying to detect stochastic signals with known covariance matrix but with no a priori knowledge on their probability density function, people usually try to project on the signal subspace (SVD,...). It is possible to take into account the structure (covariance) of the embedding noise: classically the SMF is used in such a point of view and in this case, a projection onto a one-dimension subspace is made. In theory this method can be proved to be equivalent to a two stages method: a whitening stage of noise followed by an SVD. Obviously, the other filters calculated with the SMF method can bring information for detection (or filtering, reconstruction, ...) and it is tempting to use several filters to improve the results. Such an approach has been already adopted: people naturally use the filters corresponding to the largest eigenvalues. This position comes down to project onto a subspace of dimension greater than 1. However, there is no guarantee that the signal-to-noise ratio is maximum in the subspace spanned by this vectors.

In this paper, we calculate a set of optimal filters in the sense that they maximize the signal-to-noise ratio in a subspace of dimension chosen a priori. This subspace is not necessarily those spanned by the vectors calculated by the SMF.

Prospects of applications of the CSMF can easily be imagined in image processing or stochastic transient signals detection or estimation (acoustic signals), and a extension to the classification problem can be envisaged.

\section{REFERENCES}

[1] H.L. Van Trees, Detection, Estimation and Modulation Theory, Radar-Sonar Signal Processing and Gaussian Signals in Noise, Wiley, Part III.

[2] A. Hero, Signal Detection and Classification, in 'The Digital Signal Processing Handbook' by Vijay K. Madisetti. CRC Press LLC, Series: Electrical Engineering Handbook, 1997

[3] T. Kailath, H.V. Poor, Detection of Stochastic Processes, IEEE Transactions on Information Theory, Vol.44, pp.2230-2259, n ${ }^{\circ}$ 6, October 1998.

[4] S. Verdu, H.V. Poor, Signal Selection for Robust Matched Filtering, IEEE Trans. on Communications, Vol.COM-31, pp.667-670, n ${ }^{\circ}$ 5, May 1983.

[5] S. Verdu, H.V. Poor, Minimax Robust Discrete-time Matched Filters IEEE Trans. Communications, vol. COM-31, no. 2, pp.208-215, Feb. 1983.

[6] J.G. Proakis, Digital Communications, McGraw-Hill, Inc., third edition, 1995.

[7] Y.C. Eldar, A.V. Oppenheim, Orthogonal matched filter detection, Proceedings on the International Conference Acoustics, Speech, Signal Processing (ICASSP-2001) Salt-Lake, UT, May 2001.

[8] J.F. Cavassilas, B.Xerri, Extension de la notion de filtre adapté. Contribution à la détection de signaux courts en présence de termes perturbateurs, Revue Traitement du Signal Volume 10 n $^{\circ} 3,1993$, pp.215-221.

[9] R.O. Duda, P.E. Hart, D.G. Stork Pattern Classification, John Wiley \& sons, Inc.- Interscience publication, second edition, 2001.

[10] K. Fukunaga, introuduction to Statistical Pattern Recognition, Academic Press, second edition, 1990.

[11] D. Lindgren, Subspace selection techniques for classification problems Thesis, Linköping University, Sweeden, 2002.

[12] P.-A. Absil, R. Mahony, R. Sepulchre, P.Van Dooren A Grassmann-Rayleigh quotient iteration computing invariant subspaces SIAM Review, vol. 44, no. 1, pp.5773, 2002.

[13] J. Brandts, A comparison of subspace methods for Sylvester equations Universiteit Utrecht, Matematics Insitute, preprint n1183, March 2001. http://www.library.uu.nl/digiarchief/dip/dispute/20010627-111059/1183.pdf 